\title{
Cálculo de atenuación por lluvia usando el modelo DAH y diámetros de antena para Banda Ka en México
}

\author{
S. Landeros-Ayala, R. Neri-Vela, H. Cruz-Sánchez, H. Hernández-Bautista \\ Facultad de Ingeniería, UNAM \\ E-mails:land@fi-b.unam.mx.hugo.cruz@correo.unam.mx,horacio.hb@correo.unam.mx
}

(recibido: agosto de 2001; aceptado: octubre de 2001)

Resumen

En los últimos años, el auge en la demanda de servicios de comunicación por satélite ha provocado la saturación en el uso de las frecuencias correspondientes a las bandas C y Ku. Debido a esta razón, se han buscado alternativas viables para poder satisfacer la demanda actual, así como la demanda futura, para la cual se espera un incremento considerable. Una de estas alternativas es el uso de la Banda Ka $(20 \mathrm{GHz} / 30 \mathrm{GHz})$, de ahí la importancia del estudio sobre los efectos de propagación que se experimentan a estas frecuencias, en especial, el efecto de atenuación por lluvia, ya que en este caso resulta ser significativa. El presente artículo tiene como finalidad describir el uso del Modelo DAH (cuyos autores son Asoka Dissanayake, Jeremy Allnutt y Fatim Haidara), combinado con los mapas globales de distribución de lluvia de Crane, para el cálculo de la atenuación por lluvia en sistemas de comunicación por satélite que operen en la Banda Ka. Además, se proponen diámetros de antena para los sistemas de comunicaciones en Banda Ka en diferentes localidades de la República Mexicana, empleando para ello, los márgenes de atenuación por lluvia obtenidos a través del Modelo DAH, y usando como referencias las características del satélite de comunicaciones ANIK F2 y de una estación terrena VSAT.

Descriptores: atenuación por lluvia en Banda Ka, comunicaciones por satélite, cálculos de enlace, modelo DAH, antenas en Banda Ka.

\footnotetext{
Abstract

In the last years, the peak in the demand of satellite communication services has caused the saturation in the use of the frequencies corresponding to the band, $\mathrm{C}$ and $\mathrm{Ku}$. Due to this, the engineers have looked for viable alternatives, in order to satisfy the current requisition, as well as the future demand, for which a considerable increment is expected. One of these alternatives is the use of the $\mathrm{Ka} \mathrm{Band}(20 \mathrm{GHz} / 30 \mathrm{GHz})$; that is why the importance of studying the propagation effects that are experienced at these frequencies, especially the attenuation effect by rain, as in this case, where it is significant. The present article has the purpose to describe the use of the Modelo DAH (whose authors are Asoka Dissanayake, Jeremy Allnutt and Fatim Haidara), mixed with the global maps of distribution of rain by Crane, for the calculation of the attenuation by rain in satellite communication systems operated in the $\mathrm{Ka} \mathrm{Band}$. Besides, antenna diameters for the systems of communications in $\mathrm{Ka}$ Band in different locations of the Mexican Republic, using for it the attenuation margins for rain obtained through the Modelo DAH, and using as references the characteristics of the ANIK
} 
DOI: http://dx.doi.org/10.22201/fi.25940732e.2002.03n1.002

F2 satellite and a terrestrial station VSAT, are proposed.

Keywords: rain attenuation in $\mathrm{Ka}$ Band, satellite communications, link calculations, DAH Model, Ka Band antennas.

\section{Introducción}

Debido a la congestión que presentan las bandas de frecuencia bajas como son la Banda C (4 GHz/6 $\mathrm{GHz}$ ) y la Banda $\mathrm{Ku}(12 \mathrm{GHz} / 14 \mathrm{GHz})$, la mayoría de los servicios que se ofrecen en dichas bandas se ofrecerán en la Banda $\mathrm{Ka}(20 \mathrm{GHz} / 30 \mathrm{GHz})$.

La propagación de las ondas de radio arriba de $10 \mathrm{GHz}$ a través de la atmósfera, implica no sólo a las pérdidas en el espacio libre, sino a muchos otros factores importantes. Entre estos factores tenemos los siguientes:

a) La contribución de gases de la atmósfera homogénea, debido a mecanismos de polarización resonante y no resonante.

b) La contribución de las no homogeneidades en la atmósfera.

c) La particular contribución debida a la lluvia, las nubes, la niebla, y partículas sólidas suspendidas en el aire (arena, polvo y humo) (Liebe, 1969).

Uno de los problemas que trae consigo el uso de la Banda Ka es el incremento de los fenómenos de atenuación de las ondas electromagnéticas con respecto a otras frecuencias menores. Fenómenos como la atenuación por lluvia, hacen que el diseño de un enlace en Banda Ka sea más complejo. Por ello, se hace necesario el cálculo y la predicción lo más preciso posible, de los fenómenos de propagación, principalmente en sistemas de bajo margen, con la finalidad de hacerlos más eficientes en funcionamiento y costo. De ahí la importancia del estudio del efecto de atenuación por lluvia para la Banda Ka. (Landeros et al., 1997).

\section{Desarrollo}

Modelos de atenuación por lluvia

A raíz de los datos recopilados por el ACTS (Advanced Communication Technology Satellite, lanzado en
Septiembre de 1993) y los resultados arrojados de los experimentos de propagación en la Banda Ka, se ha hecho posible la comparación de dichos resultados experimentales con resultados teóricos provenientes de diversos métodos de predicción de atenuación. Asimismo, se han buscado mejorar los modelos ya existentes para hacerlos más precisos, además de elaborar otros nuevos modelos. Uno de estos modelos es el propuesto por Asoka Dissanayake, Jeremy Allnutt y Fatim Haidara, el cual es conocido como modelo DAH. Este arroja resultados más precisos en cuanto al cálculo de atenuación por lluvia y por otros fenómenos en Banda Ka. Un estudio realizado por Robert K. Crane y Asoka Dissanayake, compara cuatro modelos: el modelo global, el de dos componentes, el recomendado por la UIT y el modelo DAH. Estos modelos fueron combinados con tres diferentes modelos de distribución de lluvia: el modelo Crane-Global, el modelo de la UIT y el modelo Rice-Holmberg. Este estudio demuestra que el único modelo que arrojó predicciones aceptables fue el modelo DAH, combinado ya sea con el modelo de distribución Global de Crane o con el modelo Rice-Holmberg. En ambos casos se obtuvo un comportamiento mucho mejor que con el uso de los modelos de Crane o con el modelo UIT para el cálculo de atenuación. Sin embargo, el modelo Rice-Holmberg utiliza datos locales que sólo pueden ser obtenidos por medio de observaciones directas durante períodos de tiempo largos (Rice y Holmberg, 1973). Aunado a esto, los resultados que arrojó este estudio dejan ver que estadísticamente los resultados que se obtienen cuando se utiliza la distribución Global de Crane son mejores que cuando se usa el modelo RiceHolmberg. A partir de las comparaciones que se realizaron con los datos recopilados con el ACTS, así como de la observación de resultados de los distintos modelos se concluyó que, de todos los modelos utilizados, el modelo Global de distribución de lluvia combinado con el modelo de predicción de atenuación DAH fue el mejor (Crane y Dissanayake, 1997). 
Para la evaluación de resultados, se realizaron diferentes pruebas de hipótesis a los modelos de atenuación combinados con los modelos de distribución de lluvia, en cuanto a los resultados que arrojaron en la predicción de atenuación en Banda $\mathrm{Ka}$, comparando los resultados que se obtuvieron de los experimentos con el ACTS. Una prueba fue con base a la desviación RMS. La prueba de hipótesis con un nivel de significancia de 0.1 , permite rechazar cualquier combinación de modelos, cuya desviación RMS sea mayor a 0.33 , y que no sea consistente con las mediciones del ACTS (Tabla 1). Las diferencias compuestas promedio, fueron utilizadas para realizar una segunda prueba de hipótesis. Un modelo es consistente con las observaciones realizadas a un nivel de significancia de 0.1 , si la magnitud de la diferencia promedio es menor a 0.07 (Tabla 2) (Rice y Holmberg, 1973).

Tabla 1. Valores de desviación RMS para diferentes modelos

\begin{tabular}{cccc}
\hline \multicolumn{4}{c}{ Datos de atenuación a 20.2 GHz } \\
\hline Modelo de atenuación & \multicolumn{3}{c}{ Modelo de distribución de lluvia } \\
\hline DAH & Global de Crane & ITU-R & Rice-Holmberg \\
\hline ITU-R & 0.28 & 0.45 & 0.32 \\
Dos componentes & 0.42 & 0.51 & 0.50 \\
Global & 0.60 & 0.63 & 0.37 \\
& 0.47 & 0.61 & 0.52 \\
\hline Modelo de atenuación & Datos de atenuación a 27.5 GHz & Rice-Holmberg \\
\hline DAH & \multicolumn{4}{c}{ Modelo de distribución de lluvia } \\
ITU-R & Global de Crane & ITU-R & 0.35 \\
Dos componentes & 0.21 & 0.51 & 0.45 \\
Global & 0.31 & 0.53 & 0.40 \\
\hline
\end{tabular}

Tabla 2. Valores de diferencia promedio compuesta para diferentes modelos

Datos de atenuación a $20.2 \mathrm{GHz}$

\begin{tabular}{cccc}
\hline Modelo de atenuación & \multicolumn{3}{c}{ Modelo de distribución de lluvia } \\
\hline & Global de Crane & ITU-R & Rice-Holmberg \\
\hline DAH & 0.14 & 0.10 & -0.06 \\
ITU-R & 0.16 & 0.12 & -0.06 \\
Dos componentes & 0.40 & 0.31 & -0.07 \\
Global & 0.30 & 0.20 & 0.30 \\
\hline & Datos de atenuación a 27.5 GHz & Rice-Holmberg \\
\hline Modelo de atenuación & \multicolumn{4}{c}{ Modelo de distribución de lluvia } & -0.20 \\
\hline DAH & Global de Crane & ITU-R & -0.14 \\
ITU-R & -0.01 & 0.06 & -0.08 \\
Dos componentes & 0.07 & 0.17 & 0.42 \\
Global & 0.32 & 0.37 & 0.27 \\
\hline \hline
\end{tabular}


Obsérvese cómo la combinación del modelo DAH con el modelo global de Crane de distribución de lluvia es el que se desempeña mejor en ambas frecuencias de experimentación y en ambas pruebas de hipótesis.

\section{Modelo DAH}

El modelo DAH (Dissanayake, Allnutt, Haidaral) es formalmente un procedimiento para predecir los efectos combinados de atenuación por lluvia, además de varios factores que contribuyen a la atenuación en un enlace de comunicación satelital. La importancia de predecir con la mayor exactitud posible la atenuación por lluvia, así como la atenuación por otros fenómenos, radica en la calidad del desempeño de un sistema de comunicaciones y la posibilidad de disminuir costos en cuanto al diseño y a la implementación de estos.

Los datos que se requieren para el modelo son:

- Latitud de la estación terrena ф (grados)

- Altitud de la estación terrena sobre el nivel del $\operatorname{mar}_{\mathrm{s}}(\mathrm{km})$

- Tasa de la intensidad de lluvia al $0.01 \%$ del promedio anual $R_{0.01}(\mathrm{~mm} / \mathrm{h})$

- Porcentaje de probabilidad de exceso para el cual se va a calcular la atenuación $p$

- Ángulo de elevación $\theta$ (grados)

- Frecuencia $f(\mathrm{GHz})$

- Ángulo de polarización $\xi$ (grados)

- Radio efectivo de la Tierra $R_{e}=8500 \mathrm{~km}$

Paso 1. Calcular la altura de congelamiento durante lluvia $h_{\mathrm{fr}}(\mathrm{km})$, a partir del valor absoluto de la latitud de la estación:

$$
\begin{array}{ll}
h_{f r}=5 & \text { para } 0^{\circ} \leq \phi<23^{\circ} \\
h_{f r}=5-0.075(\phi-23) & \text { para } \phi \geq 23^{\circ}
\end{array}
$$

Paso 2. La trayectoria inclinada $L_{s}$ bajo la altura de congelamiento es obtenida de:

$$
\mathrm{L}_{\mathrm{s}}=\frac{h_{\mathrm{fr}}-h_{\mathrm{s}}}{\operatorname{sen} \theta}(\mathrm{km})
$$

Paso 3. La proyección horizontal de la trayectoria inclinada es calculada de:

$$
\mathrm{L}_{g}=\mathrm{L}_{\mathrm{s}} \cos \theta(\mathrm{km})
$$

Paso 4. Se obtiene la intensidad de lluvia $R_{0.01}$ $(\mathrm{mm} / \mathrm{h})$ excedida para $0.01 \%$ de un promedio anual y se calcula la atenuación específica $\gamma(\mathrm{dB} / \mathrm{km})$ usando los coeficientes ampliamente publicados, dependientes de la polarización y de la frecuencia (CCIR, 1992) e ( ITU, 1995).

$$
\gamma=k\left(R_{0.01}\right)^{\alpha}(d B / k m)
$$

Paso 5. Calcular el factor de ajuste horizontal $r h_{0.01}$ para $0.01 \%$ del tiempo

$$
r h_{0.01}=\frac{1}{1+0.78 \sqrt{\frac{\mathrm{L}_{g} \gamma}{f}}-0.38\left[1-10^{\left(-2 L_{g}\right)}\right]}
$$

donde $f$ es la frecuencia en $\mathrm{GHz}$.

Paso 6. Calcular la longitud ajustada del enlace $L_{\mathrm{r}}(\mathrm{km})$ a través de la lluvia

$$
\begin{gathered}
\mathrm{L}_{r}=\frac{\mathrm{L}_{g} r h_{0.01}}{\cos \theta} \quad \text { para } \zeta>\theta \\
\mathrm{L}_{r}=\frac{h_{f r}-h_{s}}{\operatorname{sen} \theta} \quad \text { para } \zeta \leq \theta
\end{gathered}
$$

donde $\zeta=\tan ^{-1}\left(\frac{h_{f r}-h_{s}}{L_{g} r h_{0.01}}\right)$

Paso 7. Calcular el factor de ajuste vertical $r v_{0.01}$ para el $0.01 \%$ del tiempo.

$$
r_{0.01}=\frac{1}{1+\sqrt{\operatorname{sen} \theta}\left[31\left(1-e^{-\theta /(1+x)}\right) \frac{\sqrt{L_{r} \gamma}}{f^{2}}-0.45\right]}
$$

donde:

$$
\begin{array}{ll}
\chi=36-|\phi| & \text { para }|\phi|<36^{\circ} \\
\chi=0 & \text { para }|\phi| \geq 36^{\circ}
\end{array}
$$


Paso 8. La longitud efectiva $\mathrm{L}_{\mathrm{e}}(\mathrm{km})$ a través de la lluvia es:

$$
\mathrm{L}_{e}=\mathrm{L}_{r} r v_{0.01}
$$

Paso 9. La atenuación excedida en $0.01 \%$ del promedio anual es obtenida de:

$$
A_{0.01}=\gamma L_{e}(d B)
$$

Paso 10. La atenuación a ser excedida para otras indisponibilidades en el rango de $0.001 \%$ a $10 \%$ es estimada de la atenuación con indisponibilidad de $0.01 \%$ con:

$A_{p}=A_{0.01}\left(\frac{p}{0.01}\right)^{-10.655+0.033 \ln p-0.045 \ln A_{0.01}-z \operatorname{sen} \theta(1-p) \mid}$ (dB) L1. 10

donde $p$ es el porcentaje de indisponibilidad de interés y z está dada por

$$
\begin{array}{ll}
\text { para } p \geq 1 \%, z=0 & \\
\text { para } p<1 \% & \text { para }|\phi| \geq 36^{\circ} \\
z=0 & \text { para } \theta \geq 25^{\circ} \text { y }|\phi|<36^{\circ}
\end{array}
$$

$$
\begin{aligned}
& \mathrm{z}=-0.005(|\phi|-36)+1.8-4.25 \operatorname{sen} 0 \theta \\
& \text { para } \theta<25^{\circ} \text { y }|\phi|<36^{\circ}
\end{aligned}
$$

\section{Márgenes de lluvia para México en Banda Ka}

Para el cálculo de los ángulos de elevación y Azimut se consideró al satélite canadiense ANIK F-2, que estará ubicado en $111.1^{\circ}$ de longitud Oeste. Este satélite será lanzado en el año 2002 y prestará servicios en Banda Ka a Norteamérica. Se seleccionaron varias ciudades de nuestro país para obtener los márgenes de atenuación por lluvia. Estas ciudades destacan por su importancia económica además de estar ubicadas en diferentes zonas dentro del mapa global de distribución de lluvia de Crane. Los datos de cada ciudad fueron obtenidos de la base de datos del INEGI (2001).

Los resultados se muestran en la tabla 3, en donde se puede observar que la variación de la atenuación por lluvia está en función directa de la frecuencia. En las frecuencias más altas, la atenuación por lluvia se incrementa de manera significativa. Aunado a esto, la atenuación depende

\begin{tabular}{|c|c|c|c|c|c|c|c|c|c|}
\hline & \multirow[b]{2}{*}{ Disponibilidad } & \multicolumn{4}{|c|}{ Atenuación por lluvia a $29.5 \mathrm{GHz}[\mathrm{dB}]$} & \multicolumn{4}{|c|}{ Atenuación por lluvia a $20.2 \mathrm{GHz}[\mathrm{dB}]$} \\
\hline & & 99.99 & 99.9 & 99.5 & 99 & 99.99 & 99.9 & 99.5 & 99 \\
\hline Ciudad & Zona & & & & & & & & \\
\hline Xalapa-Enríquez & D3 & 45.45 & 16.51 & 7.59 & 4.91 & 23.24 & 7.88 & 3.45 & 2.18 \\
\hline Veracruz & D3 & 56.54 & 21.15 & 9.86 & 6.39 & 28.64 & 9.99 & 4.43 & 2.81 \\
\hline $\begin{array}{c}\text { México DF } \\
\text { (Cuauhtémoc) }\end{array}$ & D2 & 31.86 & 10.77 & 4.99 & 3.2 & 16.32 & 5.14 & 2.27 & 1.43 \\
\hline Guanajuato & D2 & 33.67 & 10.96 & 5.32 & 3.42 & 17.2 & 5.22 & 2.41 & 1.52 \\
\hline Guadalajara & D1 & 31.4 & 10.09 & 4.91 & 3.14 & 15.9 & 4.76 & 2.2 & 1.38 \\
\hline Puerto Vallarta & Dl & 40.26 & 13.27 & 6.6 & 4.24 & 20.18 & 6.19 & 2.93 & 1.84 \\
\hline Ensenada & $\mathrm{F}$ & 26.98 & 9.71 & 4.08 & 2.62 & 13.66 & 4.58 & 1.83 & 1.15 \\
\hline La Paz & $\mathrm{F}$ & 29.36 & 10 & 4.54 & 2.9 & 14.67 & 4.65 & 2.01 & 1.25 \\
\hline Cancún & G & 70.92 & 25.76 & 12.87 & 8.4 & 36.45 & 12.36 & 5.88 & 3.76 \\
\hline Villahermosa & G & 71.93 & 26.33 & 13.11 & 8.54 & 36.6 & 12.49 & 5.92 & 3.78 \\
\hline
\end{tabular}
del grado de disponibilidad que se requiera en el sistema de comunicaciones. Esto representa, en términos económicos, una inversión mucho más grande en un sistema que se encuentre disponible casi la totalidad del tiempo de funcionamiento, que en otro que tiene una menor disponibilidad.

Tabla 3 
El modelo DAH, en su totalidad, presenta errores menores al 20\% (Dissanayake et al., 1997). Tomando en cuenta que la variación de la lluvia que se presenta en un enlace dado es del orden de 20\% (Crane, 1993) el error para predecir la atenuación a través de un enlace de este método es comparable con este valor.

\section{Cálculo del diámetro de las antenas para Banda Ka. (Hernández y Cruz, 2001)}

Para la realización de los cálculos de enlace, se empleó una estación VSAT con las características mostradas en la tabla 4.

Se calcularon los diámetros de las antenas que se requerirían para disponibilidades de 99.99\%, 99.9\%, $99.5 \%$ y $99 \%$ para tasas de transferencia de $2.04 \mathrm{Mbps}$, $1.5 \mathrm{Mbps}, 384 \mathrm{kbps}$ y $64 \mathrm{kbps}$ en polarizaciones vertical y horizontal para los enlaces ascendentes y descendentes, respectivamente. El ancho de banda requerido, el número de portadoras por transpondedor, las condiciones que se consideraron para el enlace en cuanto a relación $\mathrm{C} / \mathrm{N}_{0}$ total y las condiciones de $\mathrm{C} / \mathrm{N}_{0}$ para los diferentes trayectos, se muestran en la tabla 5.
Considerando las diferencias para ambos enlaces, y tomando en cuenta que una estación terrena se utiliza para la transmisión y recepción simultánea en un enlace satelital, es necesario satisfacer los requerimientos mínimos para que el comportamiento del sistema sea satisfactorio. Es por esa razón, que para el diseño de una estación terrena se toma el valor máximo del diámetro. De esta forma quedan satisfechas las condiciones mínimas para el desempeño del enlace ascendente y al mismo tiempo, se satisfacen y benefician las condiciones para el enlace descendente; en otras palabras, el sistema cumplirá la disponibilidad para la cual se diseñó, para el enlace ascendente, mientras tanto tendrá una disponibilidad mayor en la sección del enlace descendente.

En cuanto a los diámetros de las antenas requeridos para establecer enlaces con niveles de disponibilidad del $99.99 \%$, se puede ver que los resultados obtenidos para las distintas ciudades de México muestran las características de una antena que son imposibles de realizar por sus grandes dimensiones y por su alto costo.

Tabla 4. Especificaciones de la estación VSAT $20 / 30 \mathrm{GHz}$

\begin{tabular}{|c|c|c|}
\hline Parámetro & Valor & Comentario \\
\hline Rango de frecuencias de $T x$ & $29.5-30 \mathrm{GHz}$ & \\
\hline Rango de frecuencias de $R x$ & $19.7-20.2 \mathrm{GHz}$ & \\
\hline Polarización Tx & Vertical & \\
\hline Polarización Rx & Horizontal/Nertical & \\
\hline Ganancia de la antena Tx/Rx@29.5 GHz & $51 / 48 \mathrm{~dB}$ & \\
\hline PIRE@1dB 25ㄷ & $53 \mathrm{dBW}$ & Con antena de $1.5 \mathrm{~m}$ \\
\hline Amplificador de Alta Potencia & $32 \mathrm{dBm}$ & \\
\hline $\mathrm{G} / \mathrm{T}$ mínima $\mathrm{Rx}$ & $24 \mathrm{~dB} / \mathrm{K}$ & Con antena de $1.5 \mathrm{~m}$ \\
\hline Figura de Ruido del Receptor @ 25ㄷ & 2.2 dB máx. & \\
\hline
\end{tabular}

Tabla 5. Condiciones para el enlace

\begin{tabular}{|c|c|c|c|c|}
\hline Tasa de transmisión & $2.04 \mathrm{Mbps}$ & $1.5 \mathrm{Mbps}$ & $384 \mathrm{kbps}$ & $64 \mathrm{kbps}$ \\
\hline Ancho de Banda (BPSK) (MHz) & 4.90 & 3.60 & 0.922 & 0.154 \\
\hline No. portadoras & 11 & 15 & 60 & 362 \\
\hline $\mathrm{C} / \mathrm{N}_{0}$ Total $[\mathrm{dBHz}]$ & 69.40 & 68.06 & 62.14 & 54.36 \\
\hline $\mathrm{C} / \mathrm{N}_{0}$ Ascendente $[\mathrm{dBHz}]$ & 75.74 & 74.40 & 68.10 & 59.99 \\
\hline $\mathrm{C} / \mathrm{N}_{0}$ Descendente $[\mathrm{dBHz}]$ & 71.40 & 70.06 & 64.14 & 56.36 \\
\hline $\mathrm{C} / \mathrm{N}_{0}$ Intermodulación $[\mathrm{dBHz}]$ & 78.04 & 76.70 & 71.53 & 64.56 \\
\hline
\end{tabular}


Las disponibilidades típicas que se emplean para el diseño de los sistemas en Banda Ka están entre 99.5\% y $99.7 \%$ (Grami et al.). En algunos sitios se pueden obtener disponibilidades mayores, dependiendo principalmente del clima del lugar, así como de la velocidad de transmisión que se desee emplear. Esto puede ser observado en los cálculos arrojados para la disponibilidad de $99.9 \%$.

Vale la pena recordar que en el diseño de un sistema se deben tomar en cuenta varios factores. El factor de la calidad en el enlace es importante, además de la disponibilidad deseada, dependiendo del servicio que se prestará; sin embargo, el factor económico es determinante en la mayoría de los casos.

En la tabla 6 se muestra un resumen de los diámetros requeridos para las estaciones terrenas por zona para satisfacer disponibilidades de $99.8 \%$ y $99.5 \%$ y $99 \%$ a partir de los resultados obtenidos de varias ciudades de la Republica Mexicana.
Como se ve en la tabla 6 , se pueden realizar disponibilidades del orden de $99.5 \%$; sin embargo, para algunas zonas geográficas con tasas bajas de lluvia esta disponibilidad puede ser mejorada, así como para zonas con tasas de lluvia altas, esta disponibilidad puede ser alcanzada con una mayor inversión en el equipo.

El modelo empleado para los cálculos de atenuación por lluvia en Banda Ka fue validado con los resultados experimentales con el ACTS por diversos investigadores de Estados Unidos a través de varios estudios. En la figura 1 se muestra el comportamiento de la predicción de atenuación por lluvia que se obtuvo a partir del modelo DAH para Ciudad Mante, Tamaulipas a $27.5 \mathrm{GHz}$. Esta localidad se encuentra ubicada en $22.75^{\circ}$ de latitud norte y $98.97^{\circ}$ de longitud Oeste a 80 metros sobre el nivel del mar y en la zona G2 del mapa de distribución de lluvia del modelo Global de Crane.

Tabla 6. Diámetros requeridos en las antenas de las estaciones terrenas por zona a diferentes disponibilidades

\begin{tabular}{cccccccccccccc}
\hline \hline & \multicolumn{9}{c}{$99.8 \%$} & \multicolumn{1}{c}{$99.5 \%$} & \multicolumn{3}{c}{$99 \%$} \\
\hline \multirow{2}{*}{ Zona } & 2.04 & 1 & 384 & 64 & 2.04 & 1 & 384 & 64 & 2.04 & 1 & 384 & 64 \\
& Mbps & Mbps & kbps & kbps & Mbps & Mbps & kbps & kbps & Mbps & Mbps & kbps & kbps \\
\hline D3 & 4.2 & 3 & 1.7 & 0.7 & 2 & 1.5 & 1 & 0.3 & 1.3 & 1 & 0.60 & 0.2 \\
D2 & 3 & 2 & 1.2 & 0.5 & 1.5 & 1 & 0.6 & 0.3 & 1.1 & 0.8 & 0.5 & 0.2 \\
D1 & 2.3 & 1.6 & 1 & 0.4 & 1.4 & 1 & 0.6 & 0.2 & 1 & 0.7 & 0.45 & 0.2 \\
F & 1.5 & 1 & 0.6 & 0.3 & 1 & 0.75 & 0.45 & 0.2 & 0.90 & 0.60 & 0.40 & 0.2 \\
G & 8 & 5.7 & 3.4 & 1.3 & 3 & 2 & 1.20 & 0.5 & 1.70 & 1.20 & 0.70 & 0.3 \\
\hline
\end{tabular}

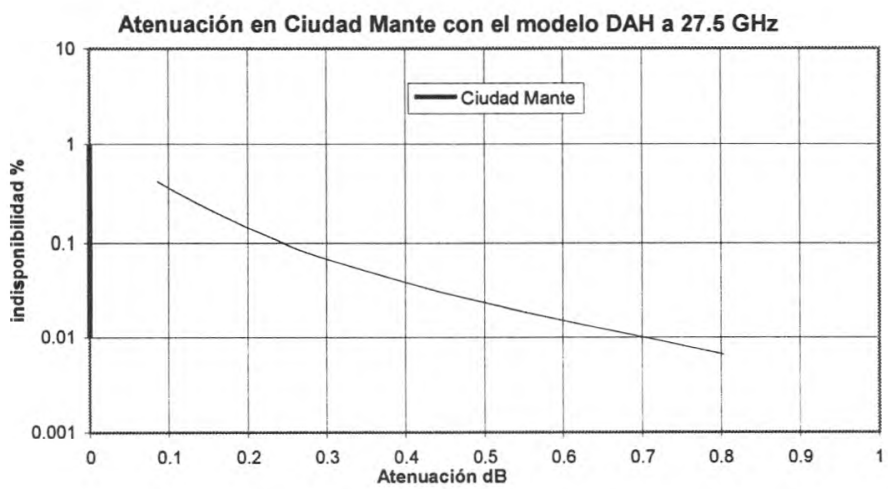

Figura1. Atenuación en Ciudad Mante, Tamaulipas con el modelo DAH a $27.5 \mathrm{GHz}$ 


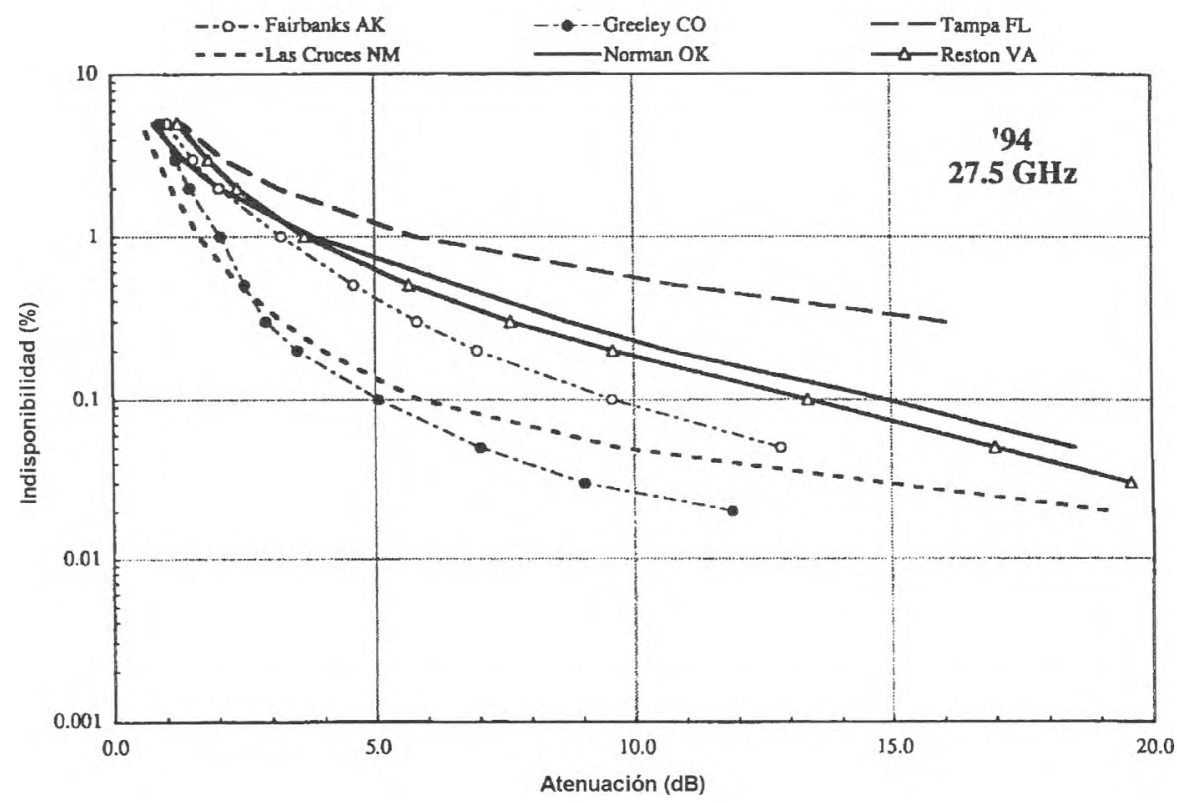

Figura 2. Atenuación por lluvia a 27.5 GHz. Datos recopilados con el ACTS

Este municipio cuenta con características de altura y zona climática idénticas a las que tenía la estación de Reston, VA, en los Estados Unidos y que fue empleada para la recopilación de datos durante el experimento de propagación del ACTS junto con otras seis estaciones ubicadas en diferentes sitios. En la figura 2, se muestran los valores de atenuación por lluvia provenientes de mediciones directas con el ACTS.

Como se podrá observar, comparando la figura 1 con la curva correspondiente a Reston, VA en la figura 2, no existe una diferencia significativa entre los valores medidos con los valores predichos para Ciudad Mante, Tamaulipas. Las diferencias existentes pueden ser atribuibles a la variación natural de la precipitación y a la diferencia entre los ángulos de elevación necesarios para el enlace con el ACTS, producida por la diferencia en cuanto a latitud y longitud

\section{Conclusiones}

El modelo que mejor predice la atenuación por lluvia en la Banda $\mathrm{Ka}$ es el modelo DAH. Esto fue comprobado en diferentes estudios con el uso de los datos que se recopilaron con el ACTS a estas frecuencias, a través de distintos procedimientos de evaluación. Fue este modelo el que se empleó para el cálculo de la atenuación por lluvia en nuestro país.
Los datos sobre atenuación por lluvia que se obtuvieron estuvieron basados en los datos de distribución de lluvia del Modelo Global de Crane. Las zonas establecidas son amplias y no son tan precisas como se hubiera deseado. Estos datos se pueden mejorar si se realiza un estudio para establecer zonas de distribución de lluvia más precisas para México.

El diámetro requerido para el enlace de recepción. siempre es menor que el requerido para el enlace de transmisión en un grado de disponibilidad dado, debido a la diferencia de los márgenes de atenuación por lluvia, los cuales están en función de las diferentes frecuencias de trabajo. Para establecer las características de una antena es necesario tomar en cuenta el diámetro mayor, ya que con esto se garantiza el funcionamiento del enlace de transmisión de la antena en la disponibilidad deseada y se garantiza el funcionamiento del enlace de recepción a disponibilidades más altas que el establecido para el primero.

Con los resultados obtenidos, se concluye que el orden de las disponibilidades de los enlaces deben estar en el orden de $99.5 \%$ a $99 \%$. En disponibilidades de $99.5 \%$, el diámetro de las antenas es pequeño en las regiones menos lluviosas de nuestro país y del orden de 2 y 3 metros para regiones lluviosas con tasas de transmisión de $2.04 \mathrm{Mbps}$. Los diámetros de las antenas que se proponen para las diferentes 
regiones y disponibilidades son los mostrados en la tabla 7 .

Tabla 7. Diámetros de antena para Banda Ka en México

\begin{tabular}{|c|c|c|c|c|c|c|c|c|c|c|c|c|}
\hline \multirow{4}{*}{ Zona } & \multicolumn{4}{|c|}{$99.8 \%$} & \multicolumn{4}{|c|}{$99.5 \%$} & \multicolumn{4}{|c|}{$99 \%$} \\
\hline & \multicolumn{4}{|c|}{ Diámetro $(\mathrm{m})$} & \multicolumn{4}{|c|}{ Diámetro (m) } & \multicolumn{4}{|c|}{ Diámetro (m) } \\
\hline & 2.04 & 1 & 384 & 64 & 2.04 & 1 & 384 & 64 & 2.04 & 1 & 384 & 64 \\
\hline & Mbps & Mbps & kbps & kbps & Mbps & Mbps & kbps & kbps & Mbps & Mbps & kbps & kbps \\
\hline D3 & 4 & 3 & 2 & 1 & 2 & 1.5 & 1 & 0.3 & 1.5 & 1 & 0.6 & 0.3 \\
\hline D2 & 3 & 2 & 1.5 & 0.6 & 1.5 & 1 & 0.6 & 0.3 & 1 & 1 & 0.5 & 0.3 \\
\hline D1 & 2 & 1.5 & 1 & 0.5 & 1.5 & 1 & 0.6 & 0.3 & 1 & 1 & 0.50 & 0.3 \\
\hline $\mathrm{F}$ & 1.5 & 1 & 0.6 & 0.3 & 1 & 1 & 0.5 & 0.3 & 1 & 0.6 & 0.5 & 0.3 \\
\hline$G$ & 8 & 6 & 3.5 & 1.5 & 3 & 2 & 1.5 & 0.5 & 2 & 1.5 & 1 & 0.3 \\
\hline
\end{tabular}

Hay que tomar en cuenta que los diámetros establecidos para las diferentes disponibilidades en la tabla anterior representan una disponibilidad mayor en el enlace descendente. Sin embargo, el funcionamiento del enlace completo estará regido por los valores enlistados.

Por último, concluimos que es factible el establecer enlaces en Banda Ka en México con la utilización de antenas pequeñas.

\section{Agradecimientos}

Asoka Dissanayake. Por proporcionarnos las correcciones sobre su artículo. (Dissanayake et al., 1997)

\section{Referencias}

Crane R.K. (1993). Estimating Risk of Earth-Satellite Attenuation Prediction. IEEE Proc., Vol. 81, 905-913.

Crane R.K. y Dissanayake A.W. (1997). ACTS Propagation Experiment: Attenuation Distribution Observations and Prediction Model Comparisons. IEEE Proc., Vol. 85, No. 6, 879-892.

Dissanayake A.W., Allnutt J.E.y Haidara F. (1997). A Prediction Model that Combines Rain
Attenuation and Other Impairments Along Earth-Space paths. IEEE Trans. Antennas Propagation, Vol. 45, No. 10, 1547-1558.

Grami A., Gordon K., and Shoamanesh A. (?). Anik F2 Ka-Band system: High-speed Internet Access, Telesat. Canadá.

www.telesat.ca/news/speeches/00-02.html.

Hernández H. y Cruz H. (2001). Cálculos de enlace y atenuación por lluvia para comunicaciones por satélite en la Banda Ka para México. Tesis de Licenciatura en Ingeniería, Universidad Nacional Autónoma de México. Ciudad Universitaria, México. INEGI (2001). Datos geográficos obtenidos de México. ITU Recommendation (1995). ITU-R RPN.837.

Landeros-Ayala S., Neri-Vela R. y Núñez-Aceves I. (1997). Cálculo de los márgenes de atenuación por lluvia para un futuro satélite mexicano en banda Ka, Ingeniería, Vol. LVII. No.4, 271-278.

Liebe H.J. (1969). Atmospheric Propagation Properties in the 10 to $75 \mathrm{GHz}$ Region: A Survey and Recommendations. ESSA Technical Report ERL 130-ITS 91. Boulder Co.

Recommendation CCIR 838 (1992). Specific Attenuation Model for Rain for Use in Prediction Methods. Rice P.L. y Holmberg N.R. (1973). Cumulative Time Statistics of Surface Point Rainfall Rates. IEEE Trans. Commun., Vol. 21, 1131-1136. 


\section{Semblanza de los autores}

Salvador Landeros-Ayala. Egresó de la Facultad de Ingeniería, UNAM, con el título de ingeniero mecánico electricista en el área de comunicaciones. Cursó la maestría en ciencias de la ingeniería en telecomunicaciones en la Universidad de Pennsylvania, Estados Unidos y obtiene el grado de doctor en ingeniería eléctrica en la Facultad de Ingeniería de la UNAM. Ha escrito artículos que se han presentado en congresos internacionales en Estados Unidos, Francia, España, Centro y Sudamérica. Fue miembro del Comité de Becas del CONACYT, director del Sistema de Satélites Nacionales y jefe de la División de Ingeniería Eléctrica. Actualmente es jefe de la División de Estudios de Posgrado de la Facultad de Ingeniería de la UNAM.

Rodolfo Neri-Vela. Cursó la carrera de ingeniero mecánico electricista y se especializó en telecomunicaciones y electrónica en la Facultad de Ingeniería, UNAM. En 1976, obtiene su maestría en telecomunicaciones en la Universidad de Essex, Inglaterra, becado por el Consejo Británico. Tres años después obtuvo el grado de doctor en radiación electromagnética aplicada por la Universidad de Birmingham, Inglaterra, como becario del Consejo Nacional de Ciencia y Tecnología (CONACYT). En 1985, se convirtió en el primer astronauta mexicano al participar en la misión 61-B de la NASA de los EU y orbitar la Tierra 109 veces. Actualmente imparte cátedra en el Departamento de Telecomunicaciones de la Facultad de Ingeniería, UNAM.

Hugo Cruz-Sánchez. Obtuvo su título como ingeniero en telecomunicaciones con mención honorífica en la Facultad de Ingeniería, UNAM. Perteneció al Programa de Alto Rendimiento Académico y ha sido consejero académico del área de Ciencias físico-matemáticas y de las ingenierías estudiante en la misma institución de 1999 al 2001. Actualmente imparte las asignaturas de procesamiento digital de señales y análisis de señales aleatorias.

Horacio Hernández-Bautista. Egresado de la Facultad de Ingeniería, UNAM como ingeniero en telecomunicaciones, obtuvo el título en el año 2001 con la tesis "Cálculos de enlace y atenuación por lluvia para comunicaciones satelitales en Banda Ka para México". Sus principales proyectos a realizar tienen que ver con la investigación, desarrollo e implementación de nuevas tecnologías en el área de las telecomunicaciones, a través de medios físicos o inalámbricos. 